\title{
New Resonances and Meson Spectroscopy at BaBar and Belle
}

\author{
Vincent Poireau \\ Laboratoire de Physique des Particules \\ F-74941 Annecy-le-Vieux - France
}

\begin{abstract}
We present a short review on the recent progresses that have been made in meson spectroscopy. We discuss the experimental discoveries made at the BaBar and Belle experiments, as well as the possible interpretations of the new resonances.
\end{abstract}

\section{Introduction}

Observation of a long list of new meson resonances has been recently reported by the BaBar and Belle experiments. We present here the new resonances observed in the $c \bar{s}$ and $c \bar{c}$ sectors.

Analyzes presented here were performed using data collected at the $\Upsilon(4 S)$ resonance with the BaBar and Belle detectors [2], located at the PEP-II and KEKB asymmetric energy $e^{+} e^{-}$ colliders.

\section{$2 \quad c \bar{s}$ mesons}

Before 2003, only four $c \bar{s}$ mesons were known: two S-wave mesons, $D_{s}\left(J^{P}=0^{-}\right)$and $D_{s}^{*}\left(1^{-}\right)$, and two P-wave mesons, $D_{s 1}(2536)\left(1^{+}\right)$and $D_{s 2}(2573)\left(2^{+}\right)$. The masses predicted by the potential model [3] were in good agreement with the measured masses. The potential model predicted also two other broad states (width of a few hundred of $\mathrm{MeV}$ ) at masses in-between $2.4-2.6 \mathrm{GeV} / c^{2}$.

\section{$2.1 \quad D_{s 0}^{*}(2317)$ and $D_{s 1}(2460)$ mesons}

In 2003, two new resonances were discovered by the BaBar and CLEO experiments: the $D_{s 0}^{*}(2317)$ and $D_{s 1}(2460)$ mesons [4]. These two resonances are very narrow, and have masses well below what was predicted by the potential model. These states are very well known experimentally: masses are measured with an error below $2 \mathrm{MeV} / c^{2}, 95 \%$ confidence level upper limits on widths are about $4 \mathrm{MeV} ; J^{P}$ quantum numbers $\left(0^{+}\right.$and $1^{+}$for $D_{s 0}^{*}(2317)$ and $D_{s 1}(2460)$ respectively), decay modes and branching fractions are also well measured. Despite a good knowledge of these states, their theoretical interpretation is still unclear. One obvious possibility is to identify these two resonances with the $0^{+}$and $1^{+}$ $c \bar{s}$ states, although it is difficult to fit these resonances within the potential model. Other interpretations have been proposed: four quark states, $D K$ molecules or $D \pi$ atoms [5].

\section{$2.2 D_{s J}^{*}(2860)$ meson}

The $D_{s J}^{*}(2860)$ resonance was discovered by BaBar in 2006 [6], looking in $c \bar{c}$ continuum: $e^{+} e^{-} \rightarrow D^{0} K^{+} X$ and $e^{+} e^{-} \rightarrow D^{+} K_{s}^{0} X$, where $X$ could be anything. A clear peak is observed in the $D K$ invariant mass, with a mass of $(2856.6 \pm 1.5 \pm 5.0) \mathrm{MeV} / c^{2}$ and a width of $(47 \pm 7 \pm 10) \mathrm{MeV}$. Given that this resonance decays to two pseudoscalars, the $J^{P}$ quantum number should be $0^{+}, 1^{-}, 2^{+}$, etc. Different interpretations have been proposed, inside the 
$c \bar{s}$ scheme: this state could be a radial excitation of the $D_{s 0}^{*}(2317)$, but other possibilities are not ruled out [7].

\section{$2.3 \quad D_{s J}(2700)$ meson}

In the same analysis, BaBar reported a broad enhancement, named $X(2690)$, at a mass of $(2688 \pm 4 \pm 3) \mathrm{MeV} / c^{2}$ and a width of $(112 \pm 7 \pm 36) \mathrm{MeV}$. A new state, the $D_{s J}(2700)$, was reported independently by Belle at a similar mass, looking at $B^{+} \rightarrow \bar{D}^{0} D^{0} K^{+}$events [8]. The study of the $D^{0} K^{+}$invariant mass reveals a clear resonance at a mass of $\left(2715 \pm 11_{-14}^{+11}\right)$ $\mathrm{MeV} / c^{2}$ with an internal width of $\left(115 \pm 20_{-32}^{+36}\right) \mathrm{MeV}$. An helicity analysis shows that the favored $J^{P}$ quantum number is $1^{-}$. Since the $X(2690)$ and $D_{s J}(2700)$ mesons have the same decay modes and that the mass and width are consistent with each other, it is reasonable to think that they are indeed the same state.

BaBar did a similar analysis [8], looking at events where $B$ decays to $\bar{D}^{(*)} D^{(*)} K$. Thanks to the many final states studied, this analysis has the advantage to be able to look at four $D^{0} K^{+}$invariant mass distributions as well as four $D^{+} K_{s}^{0}$ invariant mass distributions. Adding these final states together, a clear resonant enhancement is seen around a mass of $2700 \mathrm{MeV} / c^{2}$. Also, adding the four $D^{* 0} K^{+}$and four $D^{*+} K_{s}^{0}$ invariant mass distributions together, a similar enhancement is observed around a mass of $2700 \mathrm{MeV} / c^{2}$. No precise measurement was given by this preliminary analysis yet.

The potential model predicts the $2^{3} S_{1} c \bar{s}$ state at a mass of $2720 \mathrm{MeV} / c^{2}$. Also, from chiral symmetry considerations, a $1^{+}-1^{-}$doublet of states has been predicted. If the $1^{+}$state is identified as the $D_{s 1}(2536)$, the mass predicted for the $1^{-}$state is $2721 \pm 10$ $\mathrm{MeV} / c^{2}[9]$.

\section{$3 \quad c \bar{c}$ mesons}

\section{$3.1 \quad X(3940), Y(3940)$ and $Z(3930)$ mesons}

Three new states were discovered by Belle at masses around $3940 \mathrm{MeV} / c^{2}$ [10]. Although their mass are very close to each other, these new states are thought to be different resonances. The $X(3940)$ state was discovered in $e^{+} e^{-} \rightarrow J / \Psi X(3940)$, looking at the recoiling mass to the $J / \Psi$. The parameters of this resonance are $M=(3943 \pm 6 \pm 6) \mathrm{MeV} / c^{2}$ and $\Gamma=(15.4 \pm 10.1) \mathrm{MeV}$. This new state was also seen decaying to $D D^{*}$, but not $D D$. One possible interpretation is to identify this resonance with the unobserved $c \bar{c}$ charmonium state $\eta_{c}(3 S)\left[3^{1} S_{0}\right]$, although other interpretations have also been proposed.

A near threshold enhancement was observed by Belle in $B \rightarrow J / \Psi \omega K$, looking at the $J / \Psi \omega$ invariant mass. This resonance, called $Y(3940)$, has a mass of $(3943 \pm 11 \pm 13) \mathrm{MeV} / c^{2}$ and a width of $(87 \pm 22 \pm 26) \mathrm{MeV}$. This state could be interpreted as the $c \bar{c}$ state $\chi_{c 1}^{\prime}\left[2^{3} P_{1}\right]$.

Finally, a new resonance, the $Z(3930)$, was discovered in $\gamma \gamma \rightarrow D \bar{D}$ with a mass of $(3929 \pm 5 \pm 2) \mathrm{MeV} / c^{2}$ and a width of $(29 \pm 10 \pm 2) \mathrm{MeV}$. One possibility is to identify this resonance with the $c \bar{c}$ state $\chi_{c 2}^{\prime}\left[2^{3} P_{2}\right]$.

\section{$3.2 \quad X(3872)$ meson}

The $X(3872)$ meson was discovered by Belle [11] in $B^{ \pm} \rightarrow X(3872) K^{ \pm}$with $X(3872) \rightarrow$ $J / \psi \pi^{+} \pi^{-}$in 2003 , and quickly confirmed by the BaBar [11], CDF and D0 experiments. Its mass is known very precisely, $3871.81 \pm 0.36 \mathrm{MeV} / c^{2}$, and its width is less than $2.3 \mathrm{MeV}$ 
at $90 \%$ confidence level. This state was also observed in the final state $J / \psi \gamma[12]$, which implies that its $C$ quantum number is equal to +1 . The study of the $\pi^{+} \pi^{-}$invariant mass distribution by Belle and an angular analysis by CDF shows that $J^{P C}=1^{++}$is favored (although $2^{++}$is still possible). It has also to be noted that a search for a charged partner was performed by BaBar, but no signal was found [12].

The Belle experiment did a study of the channel $B \rightarrow \bar{D}^{0} D^{0} \pi^{0} K$ and observed a clear excess in the $\bar{D}^{0} D^{0} \pi^{0}$ invariant mass [13]. The surprise came from the measure of the mass: $3875.4 \pm 0.7_{-2.0}^{+1.2} \mathrm{MeV} / c^{2}$, which is in disagreement with the mass measured in the $X(3872) \rightarrow$ $J / \psi \pi^{+} \pi^{-}$channel. This discrepancy was confirmed by the BaBar experiment [13], looking at the $B \rightarrow \bar{D}^{0} D^{* 0} K$ channel (where both decays of $D^{* 0}, D^{0} \pi^{0}$ and $D^{0} \gamma$, are taken into account). An excess is observed in the $\bar{D}^{0} D^{* 0}$ invariant mass, with a mass of $3875.6 \pm 0.7_{-1.5}^{+1.4}$ $\mathrm{MeV} / \mathrm{c}^{2}$. The masses between Belle and BaBar are in good agreement and are $2.2 \sigma$ away from the $X(3872)$ mass in the $J / \psi \pi^{+} \pi^{-}$channel. If this excess is due to the $X(3872)$ resonance, then the quantum number $J^{P}=2^{+}$is disfavored.

The interpretation of the $X(3872)$ state is rather difficult [14] since there is no satisfactory $c \bar{c}$ assignment for this resonance. The coincidence between this resonance mass and the $\bar{D}^{0} D^{* 0}$ mass led some authors to propose that the $X(3872)$ is a bound state of the $\bar{D}^{0}$ and $D^{* 0}$ mesons with small binding energy. One of the prediction of this model is that $B^{0} \rightarrow$ $X(3872) K^{0}$ is suppressed by approximately a factor 10 compared to $B^{+} \rightarrow X(3872) K^{+}$. Experimentally, this ratio is measured to $0.50 \pm 0.30 \pm 0.05$ in the $X(3872) \rightarrow J / \psi \pi^{+} \pi^{-}$ channel and to $2.23 \pm 0.93 \pm 0.55$ in the $B \rightarrow \bar{D}^{0} D^{* 0} K$ channel. It has also been proposed that the $\mathrm{X}(3872)$ resonance is a four quark state. In this case, the model predicts two neutral states and two charged states, with a difference of mass between the two neutral states (produced respectively in $B^{0}$ and $B^{+}$decays) of $(7 \pm 2) \mathrm{MeV} / c^{2}$. The experimental results show a mass difference of $(2.7 \pm 1.3 \pm 0.2) \mathrm{MeV} / c^{2}$ in the $X(3872) \rightarrow J / \psi \pi^{+} \pi^{-}$ channel and $(0.2 \pm 1.6) \mathrm{MeV} / c^{2}$ in the $B \rightarrow \bar{D}^{0} D^{* 0} K$ channel. Other possibilities have been mentioned like glueball or hybrid state.

\section{$3.3 \quad Y(4260)$ meson}

The $Y(4260)$ state constitutes also quite a mystery. This new state, with $J^{P C}=1^{--}$, was discovered by BaBar in $e^{+} e^{-} \rightarrow \gamma_{I S R}\left(J / \psi \pi^{+} \pi^{-}\right)$, with a photon radiated in the initial state [15]. This resonance was confirmed by Belle [15] and CLEO, although masses disagree between experiments. BaBar measures $M=(4259 \pm 8) \mathrm{MeV} / c^{2}$ and $\Gamma=(88 \pm 23) \mathrm{MeV}$, Belle measures $M=\left(4295 \pm 10_{-3}^{+10}\right) \mathrm{MeV} / c^{2}$ and $\Gamma=\left(133_{-22-6}^{+26+13}\right) \mathrm{MeV}$ while CLEO measures $M=\left(4283_{-16}^{+17} \pm 4\right) \mathrm{MeV} / c^{2}$. A $3 \sigma$ enhancement was also reported by BaBar in $B \rightarrow$ $Y(4260) K^{-}$, followed by $Y(4260) \rightarrow J / \psi \pi^{+} \pi^{-}$[15], although this result needs confirmation by other experiments. Searches for this resonance were performed in other channels $\left(e^{+} e^{-} \rightarrow\right.$ $\left.\gamma_{I S R}(D \bar{D}), e^{+} e^{-} \rightarrow \gamma_{I S R}\left(\Phi \pi^{+} \pi^{-}\right), e^{+} e^{-} \rightarrow \gamma_{I S R}(p \bar{p}), e^{+} e^{-} \rightarrow \gamma_{I S R}(J / \psi \gamma \gamma)\right)$, but no positive results were reported [15].

One of the surprise concerning this resonance came from the search of the $Y(4260)$ going to the decay mode $\psi(2 S) \pi^{+} \pi^{-}$in ISR production [16]. A clear signal is observed in this channel, however with a mass measurement incompatible with the previous BaBar result. The mass found in this channel is $(4234 \pm 24) \mathrm{MeV} / c^{2}$ with a width of $(172 \pm 33) \mathrm{MeV}$. This measurement, although incompatible with the BaBar measurement in the $J / \psi \pi^{+} \pi^{-}$ channel, is compatible with the Belle measurement. More data and experiments looking at this channel are needed to be able to conclude if this excess is due to the $Y(4260)$. 
The interpretation of this state is far from obvious [17]. There is no $c \bar{c}$ assignment for a $1^{--}$state of this mass. This is also probably not a glueball, since in this case we would have expected a decay to $\Phi \pi^{+} \pi^{-}$, which was not observed. Other possibilities are four quark state $[c s][\bar{c} \bar{s}]$, hybrid meson or $\omega \chi_{c 1}$ molecule.

\section{Conclusion}

Although no new resonances were discovered in many years, BaBar and Belle gave an impressive list of new results since 1999. In the $c \bar{s}$ sector, the $D_{s 0}^{*}(2317)$ and $D_{s 1}(2460)$ mesons are now very well known experimentally, but no definite interpretation was given theoretically. The $D_{s J}^{*}(2860)$ and $D_{s J}(2700)$ mesons were discovered recently and need more experimental inputs. In the $c \bar{c}$ sector, it seems plausible to identify the $X(3940), Y(3940)$ and $Z(3930)$ mesons to charmonium states, although other explanations have been proposed. The $X(3872)$ and $Y(4260)$ resonances are not charmonium states, and thus are probably the first occurrences of non standard quark content.

A lot of analyzes are still in progress with the current data set in BaBar and Belle: more decay modes for the resonances presented here are being investigated. These two experiments are taking data until the end of 2008 , which is the promise of more surprises to arise.

The author is very grateful to the organizers of the DIS 2007 conference for their support and all efforts in making this venue successful.

\section{References}

[1] Slides: http: //indico. cern. ch/contributionDisplay py? contribId=202\&sessionId=5\&conf Id=9499

[2] BaBar Coll., Nucl. Instrum. Meth. A 4791 (2002) ; Belle Coll., Nucl. Instrum. Meth. A 479117 (2002).

[3] S. Godfrey and N. Isgur, Phys. Rev. D 32189 (1985).

[4] Belle Coll., Phys. Rev. Lett. 91 262001(2003) ; BaBar Coll., Phys. Rev. D74 032007 (2006).

[5] H-Y Cheng and W-S Hou, Phys. Lett. B566 193 (2003) ; T. Barnes, F. E. Close and H. J. Lipkin, Phys. Rev. D68 054006 (2003) ; A. Szczepaniak, Phys. Lett. B567 23 (2003).

[6] BaBar Coll., Phys. Rev. Lett. 97222001 (2006).

[7] E. van Beveren and G. Rupp, Phys. Rev. Lett. 97202001 (2006) ; P. Colangelo, F. De Fazio and S. Nicotri, Phys. Lett. B642 48 (2006).

[8] Belle Coll., arXiv:hep-ex/0608031 (2006) ; BaBar Coll., preliminary.

[9] M. A. Nowak, M. Rho and I. Zahed, Phys.Polon. B 35, 2377 (2004).

[10] Belle Coll., arXiv:hep-ex/0507019 (2005) ; Belle Coll., Phys. Rev. Lett. 94182002 (2005) ; Belle Coll., Phys. Rev. Lett. 96082003 (2006).

[11] Belle Coll., Phys. Rev. Lett. 91262001 (2003) ; BaBar Coll., Phys. Rev. D73 011101 (2006).

[12] Belle Coll., arXiv:hep-ex/0505037 (2005) ; Belle Coll., arXiv:hep-ex/0505038 (2005) ; BaBar Coll., Phys. Rev. D74 071101 (2006).

[13] Belle Coll., Phys. Rev. Lett. 97162002 (2006) ; BaBar Coll., preliminary.

[14] E. Braaten and M. Kusunoki, Phys. Rev. D71 074005 (2005) ; L. Maiani et al, Phys. Rev. D71 014028(2005).

[15] BaBar Coll., Phys. Rev. Lett. 95 142001(2005) ; Belle Coll., arXiv:hep-ex/0612006 (2006) ; BaBar Coll., arXiv:hep-ex/0607083 (2006) ; BaBar Coll., Phys. Rev. Letter D73, 011101 (2006).

[16] BaBar Coll., arXiv:hep-ex/0610057 (2006).

[17] S-L Zhu, Phys. Lett. B625 212 (2005) ; Phys. Rev. D72 031502 (2005) ; L. Maiani et al, Phys. Lett. B634 399 (2006). 